\title{
Effect of CXCR4 silencing with shRNA on MAPK signaling in ovarian cancer
}

\author{
XIULI WANG, HONGYING WANG, XIA WEI, AIXIA WANG, LINA WEN, LI WANG and QUN HUANG \\ Department of Obstetrics and Gynecology, Liaocheng People's Hospital of Shandong Province, \\ Liaocheng, Shandong 252000, P.R. China
}

Received September 21, 2015; Accepted March 9, 2017

DOI: $10.3892 / \mathrm{ol} .2018 .8550$

\begin{abstract}
Our previous study demonstrated that short hairpin RNA (shRNA) targeting of C-X-C chemokine receptor type 4 (CXCR4) significantly inhibited cell proliferation, metastasis and invasion. On the basis of these results, the aim of the present study was to determine the effects of shRNA-CXCR4 silencing on mitogen-activated protein kinase (MAPK) signaling in human SW626 ovarian cancer cells. Following silencing the CXCR4 gene with shRNA, the mRNA expression of apoptosis signal-regulating kinase 1 (ASK1) was determined using the reverse transcription-quantitative polymerase chain reaction, whereas the protein expression of extracellular-signal-regulated kinase (ERK)1/2 and phosphorylated (p)-c-Jun were determined using immunocytochemistry and western blotting. SW626 cells transfected with shRNA-CXCR4 exhibited significantly increased ASK1 mRNA expression $(\mathrm{P}<0.05)$, significantly increased $\mathrm{p}$-c-Jun protein expression $(\mathrm{P}<0.05)$, and significantly decreased ERK1/2 protein expression $(\mathrm{P}<0.05)$. Silencing the $C X C R 4$ gene with shRNA significantly inhibited cell proliferation, promoted cell apoptosis and may be mediated by the MAPK signaling pathway.
\end{abstract}

\section{Introduction}

As the most malignant type of tumor of the female reproductive system, ovarian cancer has a marked impact on the health of females (1) In total, $29 \%$ of incidences of ovarian cancer are epithelial ovarian cancer, and its mortality has ranked first among all gynecological cancers due to its hidden incidence and rapid progression (2). RNA interference (RNAi) may effectively inhibit cell proliferation, promote cell apoptosis and downregulate or silence gene expression; as such, RNAi is an attractive prospect in the genetic treatment of cancer.

Correspondence to: Dr Hongying Wang, Department of Obstetrics and Gynecology, Liaocheng People's Hospital of Shandong Province, Liaocheng, Shandong 252000, P.R. China

E-mail: hongyingwang6@163.com

Key words: ovarian cancer, C-X-C chemokine receptor type 4, RNA interference, mitogen-activated protein kinase pathway
Scotton et al (3) revealed that the expression of C-X-C chemokine receptor type 4 (CXCR4) served an important role in the targeted metastasis of ovarian cancer cells. CXCR4 silencing with RNAi significantly inhibited breast cancer cell proliferation and invasion (4). Our previous study confirmed that CXCR4-short hairpin RNA (shRNA) in human SW626 ovarian cancer cells significantly inhibited CXCR4 mRNA and protein expression, and additionally suppressed cell proliferation, metastasis and invasion (5). Since the mitogen-activated protein kinase (MAPK) signaling pathway serves a key role in the regulation of various cell functions, particularly cell proliferation, differentiation and apoptosis (6), the aim of the present study was to investigate the effects of shRNA-induced CXCR4 silencing in the MAPK signal transduction pathway.

\section{Materials and methods}

Materials and reagents. The SW626 human epithelial ovarian cancer cell line was purchased from American Type Culture Collection (Manassas, VA, USA). Other reagents included the oligonucleotide probe for $C X C R 4$, pGenesil plasmid and Escherichia coli DH5a (Wuhan Genesil Biotechnology, Wuhan, China); a one-step plasmid extraction kit (Shanghai Sangong Pharmaceutical Co., Ltd., Shanghai, China); TRIzol RNA kit [Tiangen Biotech (Beijing) Co., Ltd, Beijing, China]; Lipofectamine ${ }^{\circledR} 2000$ transfection kit (Invitrogen; Thermo Fisher Scientific, Inc., Waltham, MA, USA); primers for apoptosis signal-regulating kinase 1 (ASK1) and GAPDH (Shanghai Sangong Pharmaceutical Co., Ltd.); rabbit anti-extracellular-signal-regulated kinase (ERK)1/2 (cat. no. 9102) and rabbit anti phosphorylated (p)-c-Jun $\left(\operatorname{Ser}^{73}\right)$ (cat. no. 9164s) antibodies (Cell Signaling Technology, Inc., Danvers, MA, USA) (dilutions, 1:100); PrimeScript ${ }^{\circledR}$ RT reagent kit (Perfect Real Time) and SYBR ${ }^{\circledR}$ Premix Ex Taq ${ }^{\mathrm{TM}}$ II (Perfect Real Time) (Takara Biotechnology, Co., Ltd., Dalian, China); and a diaminobenzidine (DAB) kit (PV-9000 kit reagents 1 and 2; Beijing Zhongshan Golden Bridge Biotechnology Co., Ltd.).

Cell culture. Cells were cultured in high-glucose Dulbecco's modified Eagle's medium (Hyclone; GE Healthcare Life Sciences, Logan, MT, USA) with $10 \%$ fetal bovine serum at $37^{\circ} \mathrm{C}$ and $5 \% \mathrm{CO}_{2}$, and were digested by $0.25 \%$ trypsin or EDTA for passaging. Following the adjustment to a proper concentration, the cells were inoculated into a sterile 6-well plate. 

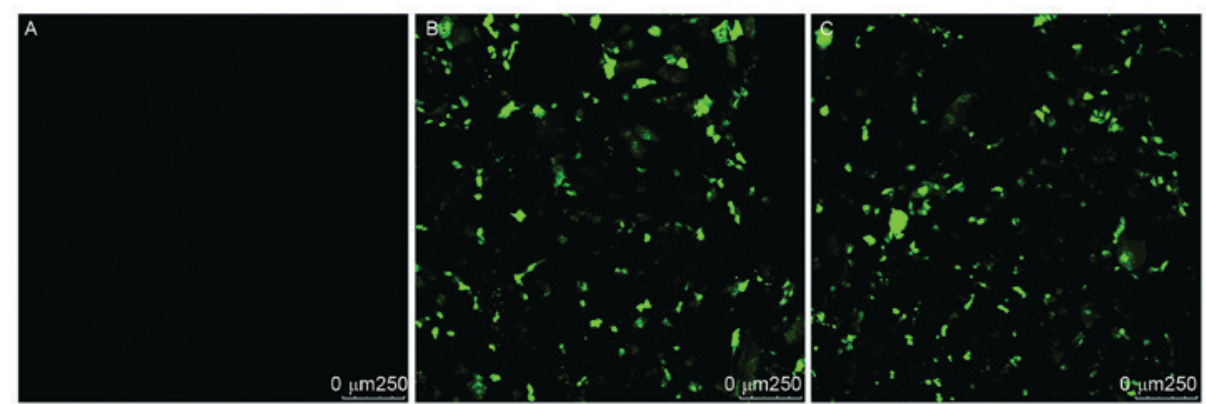

Figure 1. Transfection efficiency of C-X-C chemokine receptor type 4-short hairpin RNA in human SW626 ovarian cancer cells. (A) Blank control, (B) empty vector group, and $(\mathrm{C})$ interference group.

Plasmid transformation, extraction and purification. In our previous study, we prepared and identified a DH5 $\alpha$ strain containing the $C X C R 4(1+2+3)$ plasmid with three CXCR4 interference sequences (CXCR41, 5'-AACCCTGTTTCCGTGAAG A-3'; CXCR42, 5'-ACCATCTACTCCATCATCT-3'; CXCR43: 5-CCTCTATGCTTTCCTTGGA-3'). Cells were preserved at $-80^{\circ} \mathrm{C}$ and activated prior to use. Following cell culture and transformation, the plasmids were extracted using the one-step kit and preserved for subsequent use.

Transient cell transfection. Transient transfection was performed using Lipofectamine ${ }^{\circledR} 2000$, according to the manufacturer's protocol. For transfection, cells were randomly divided into three groups: Interference group (SW626 cells transfected with the CXCR4-shRNA vector), empty vector group (SW626 cells transfected with the blank control vector to exclude the effect of the plasmid vector on the experimental results) and the blank control group (untreated SW626 cells). The transfection was performed once the cells had reached $90 \%$ confluence in a 6 -well plate, and the transfection efficiency was observed under a fluorescence microscope after $48 \mathrm{~h}$ of culture.

Reverse transcription-quantitative polymerase chain reaction (RT-qPCR) determination of ASK1 mRNA expression. Total RNA extraction, RNA reverse transcription and cDNA synthesis were performed using the PrimeScript ${ }^{\circledR}$ RT reagent kit, according to the manufacturer's protocol. The $20 \mu \mathrm{l} \mathrm{PCR}$ reaction volume contained $2 \mu \mathrm{l}$ cDNA and $0.8 \mu 1$ primers and used SYBR ${ }^{\circledR}$ Premix Ex Taq ${ }^{\mathrm{TM}}$ II. The upstream primer sequence for ASK1 was 5'-CCAGCGTCCTAGCCAATG-3', and the downstream primer sequence was 5'-CCCTGACAG AAGAGGCACTAA-3'. The reaction conditions were $95^{\circ} \mathrm{C}$ for $30 \mathrm{sec}, 95^{\circ} \mathrm{C}$ for $5 \mathrm{sec}$ and $60^{\circ} \mathrm{C}$ for $30 \mathrm{sec}$ for a total of 40 cycles, and the fluorescent signal from the device was automatically collected. Quantification and normalization was achieved using the $2^{-\Delta \Delta \mathrm{Cq}}$ method (7). At the end of the final cycle, the melting curve was generated to verify the PCR product specificity.

Immunocytochemical determination of ERK1/2 and p-c-Jun protein expression. At $48 \mathrm{~h}$ after transfection, cells were collected and fixed with $3 \%$ paraformaldehyde (20 min), incubated with $0.5 \%$ Triton $\mathrm{X}-100$ at room temperature for $20 \mathrm{~min}$, blocked with $5 \%$ bovine serum albumin at $4^{\circ} \mathrm{C}$ for $20 \mathrm{~min}$, incubated with primary antibody (against ERK1/2 or p-c-Jun; 1:50) at $4^{\circ} \mathrm{C}$ overnight, incubated with secondary antibody at room temperature on the second day for $1 \mathrm{~h}$, washed with PBS three times, stained with DAB, mounted with $2 \%$ Mowiol, observed and images were captured under a microdissection light microscope (Leica Microsystems GmbH, Wetzlar, Germany). Image-Pro Plus software (version 6.0) was used to analyze the mean optical density (MOD), which was calculated as $\mathrm{MOD}=($ summarized integrated optical density $) /$ area and the results are presented as the mean \pm standard deviation.

Western blot determination of ERK1/2 and p-c-Jun protein expression. At $48 \mathrm{~h}$ after transfection, the cells were collected and lysed on ice by the phenylmethylsulfonyl fluoride-containing radioimmunoprecipitation solution, and $50 \mu \mathrm{g}$ protein samples were quantified using a bicinchoninic acid kit and separated by SDS-PAGE (10\% gel). The proteins were then transferred onto a nitrocellulose membrane, blocked with $5 \%$ skimmed milk at room temperature for $1 \mathrm{~h}$, incubated with the primary antibody at $4{ }^{\circ} \mathrm{C}$ overnight, washed with $0.05 \%$ Tris-buffered saline (TBS), incubated with secondary antibody at $37^{\circ} \mathrm{C}$ for $1 \mathrm{~h}$, washed by $0.05 \% \mathrm{TBS}$ again, and stained by DAB. The experiment was repeated three times, and the gray value ratio between band ERK1/2 or p-c-Jun and the internal reference ( $\beta$-actin) was considered the relative protein expression level. Each experiment was repeated for three times.

Statistical analysis. SPSS software (version 13.0; SPSS, Inc., Chicago, IL, USA) was used for the statistical analysis. Data were tested by one-way analysis of variance, and two-group comparisons were made using the least significant differences method. $\mathrm{P}<0.05$ was considered to indicate a statistically significant difference.

\section{Results}

Transient transfection efficiency of CXCR4-shRNA. The present study successfully obtainedCXCR4-shRNA-containing SW626 cells using cationic liposome-mediated transfection, and the transfection efficiency was $<80 \%$ (Fig. 1).

Effect of CXCR4-shRNA on ASK1 mRNA expression. Following cell transfection, ASK1 mRNA expression was determined by the ABI PRISM ${ }^{\circledR} 7300$ amplification system. As summarized in Table I, the ASK1 mRNA expression in the interference group was significantly different compared with that in the blank control and empty vector groups, and 
Table I. Expression of ASK1 mRNA following C-X-C chemokine receptor type 4-short hairpin RNA transfection.

\begin{tabular}{|c|c|c|c|c|c|}
\hline Gene & Group & $\mathrm{Cq}$ & $\Delta \mathrm{Cq}$ & $\Delta \Delta \mathrm{Cq}$ & P-value \\
\hline \multirow[t]{3}{*}{ ASK1 } & Blank control & $32.0329 \pm 0.8002$ & 15.5343 & 0 & \\
\hline & Empty vector & $31.5086 \pm 1.31723$ & 15.1 & -0.4343 & $0.447^{\mathrm{a}}$ \\
\hline & Interference & $29.4300 \pm 0.4452$ & 12.1329 & -3.4017 & $0.000^{\mathrm{b}}$ \\
\hline \multirow[t]{3}{*}{ GAPDH } & Blank control & $16.4986 \pm 0.4831$ & & & \\
\hline & Empty vector & $16.4086 \pm 0.4938$ & & & \\
\hline & Interference & $17.2971 \pm 0.2880$ & & & \\
\hline
\end{tabular}

ASK1, apoptosis signal-regulating kinase $1 ;{ }^{a} \mathrm{P}>0.05$, empty vector vs. blank control; ${ }^{b} \mathrm{P}<0.001$, interference vs. blank control and empty vector.

Table II. Immunocytochemical determination of extracellular signal-regulated kinase $1 / 2$ protein expression $48 \mathrm{~h}$ post-transfection.

\begin{tabular}{lcr}
\hline Group & Mean optical density & P-value \\
\hline Blank control & $71.2117 \pm 19.9886$ & $0.368^{\mathrm{a}}$ \\
Empty vector & $63.8878 \pm 2.3245$ & $0.001^{\mathrm{b}}$ \\
Interference & $27.0500 \pm 7.3917$ & $<0.001^{\mathrm{c}}$
\end{tabular}

${ }^{\mathrm{a}} \mathrm{P}>0.05$, empty vector vs. blank control; ${ }^{\mathrm{b}} \mathrm{P}<0.01$, interference vs. empty vector; ${ }^{\mathrm{C}} \mathrm{P}<0.001$, interference and blank control.

the $\Delta \mathrm{Cq}$ value of the interference group was also significantly different compared with those of the other two groups $(\mathrm{P}<0.001)$. However, no significant difference was observed between the blank control and the empty vector group $(\mathrm{P}>0.05)$.

Effect of CXCR4-shRNA transfection on ERK1/2 protein expression. Immunocytochemistry and western blotting demonstrated that compared with the blank control and empty vector groups, SW626 cells transfected with CXCR4-shRNA exhibited significantly decreased ERK1/2 protein expression levels $(\mathrm{P}<0.05)$, whereas no significant difference was observed between the blank control and empty vector groups (P>0.05) (Table II; Figs. 2 and 3).

Effect of CXCR4-shRNA transfection on p-c-Jun protein expression. Immunocytochemistry and western blotting identified that, compared with the blank control and empty vector group, SW626 cells transfected with CXCR4-shRNA exhibited significantly increased $\mathrm{p}$-c-Jun protein expression levels $(\mathrm{P}<0.01)$, whereas no significant difference was identified between the blank control and the empty vector group (P>0.05) (Table III; Figs. 4 and 5).

\section{Discussion}

MAPK is a serine/threonine protein kinase that participates in one of the most important types of signal transduction pathways in eukaryotes, and serves a key role in regulating gene expression and cytoplasmic function (8). The MAPK signaling pathway primarily includes ERKs, c-Jun N-terminal
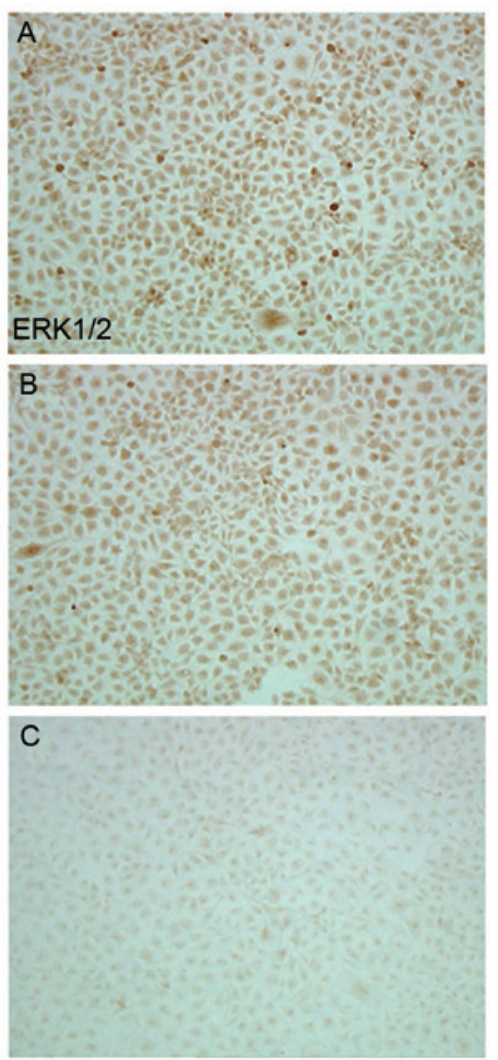

Figure 2. Immunocytochemical determination of ERK1/2 protein expression at $48 \mathrm{~h}$ after transfection. (A) Blank control, (B) empty vector group, and (C) interference group). Magnification, x100. Lane 1, 2 and 3 represent blank control, empty vector and interference groups, respectively, in the SW626 cell line. ERK1/2, extracellular-signal-regulated kinase 1/2.

kinases (JNKs) and p38 MAPK (9). The MAPK signaling pathway is involved in the regulation of various cell functions, particularly cell proliferation, differentiation and apoptosis (6). A previous study identified that, compared with normal ovarian tissues and benign ovarian lesions, MAPK activity in ovarian cancer tissues was significantly increased (10). Hayakawa et al (11) demonstrated that the MAPK was in a continuous activating status in ovarian cancer cells, and additional phosphorylation antibody detection of upstream kinase dual-specificity mitogen-activated protein kinase kinase 1 and RAF proto-oncogene serine/threonine-protein kinase (RAF)1 illustrated that MAPK activation was associated with upstream kinase activation. Inhibiting ERK1/2 activity with MAPK 
Table III. Immunocytochemical determination of phosphorylated c-Jun protein expression at $48 \mathrm{~h}$ after transfection.

\begin{tabular}{lcr}
\hline Group & Mean optical density & P-value \\
\hline Blank control & $34.9507 \pm 2.3619$ & \\
Empty vector & $37.2107 \pm 2.8655$ & $0.253^{\mathrm{a}}$ \\
Interference & $57.0649 \pm 3.5716$ & $<0.001^{\mathrm{b}}$ \\
\hline
\end{tabular}

${ }^{\mathrm{a}} \mathrm{P}>0.05$, empty vector vs. blank control; ${ }^{\mathrm{b}} \mathrm{P}<0.001$, interference vs. blank control and empty vector.

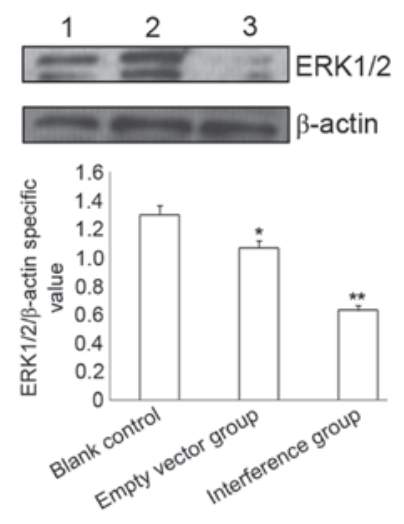

Figure 3. Western blot determination of ERK1/2 protein expression at $48 \mathrm{~h}$ after transfection $(\mathrm{P}<0.01 \mathrm{vs}$. the interference group). ERK1/2, extracellular-signal-regulated kinase $1 / 2 .{ }^{*} \mathrm{P}>0.05$, empty vector vs. lank control; ${ }^{* *} \mathrm{P}<0.001$, interference vs. blank control and empty vector.

blockers promoted the apoptosis of ovarian cancer cells, which additionally elevated cellular sensitivity to chemotherapy. The MAPK pathway realizes signal transduction via the continuous phosphorylation of MAPK kinase kinase (MAPKKK), MAPK kinase (MAPKK), and MAPK (12). Previous studies identified a number of MAPK pathways and various extracellular stimulators, including cytokines, G-protein-coupled receptors, stress signals and mitogens, may all activate various MAPK signaling transduction types. As the primary signal proteins involved in the MAPK pathway, ERK1/2, JNK and p38 exert various biological effects (13). ERK1/2 activation is primarily associated with cell proliferation, whereas JNK and p38 primarily regulate cellular apoptosis.

TheERK1/2pathway,theearliestidentifiedRas-RAF-MAPK signaling pathway (14), may be activated by a variety of growth factors or cytokines and regulates cell proliferation and differentiation, so regulating ERK activation serves a significant role in the occurrence and progression of cell proliferation (15). A previous study revealed that, upon extracellular stimulation, ERK is a key factor in determining whether a cell undergoes end-stage differentiation or apoptosis (16). ERK activation relies on signals passing from the cell-surface receptor to the nucleus via GTPase Ras, RAF1, serine/threonine kinase and MAPK/ERK kinase dual-specificity kinases (16). Another study indicated that CXCR3 may promote the metastasis of SKOV3 ovarian cancer cells via the ERK1/2 pathway, which was inhibited following ERK1/2 blockage with PD98059 (17). Tarcic and Yarden (18) suggested that the epidermal growth

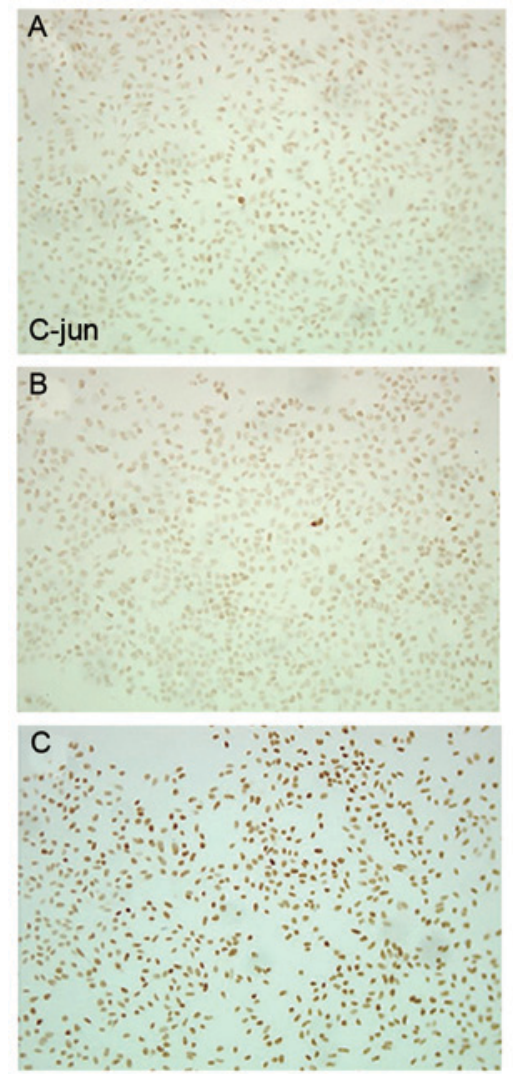

Figure 4. Immunocytochemical determination of phosphorylated c-Jun protein expression at $48 \mathrm{~h}$ following transfection. (A) Blank control, (B) empty vector group, and (C) interference group.

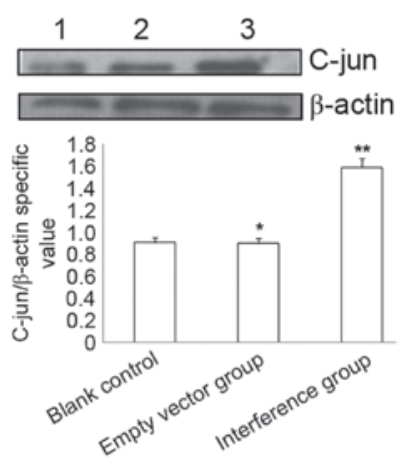

Figure 5. Western blotting determination of phosphorylated c-Jun protein expression at $48 \mathrm{~h}$ after transfection. ${ }^{*} \mathrm{P}>0.05$, empty vector vs. blank control; ${ }^{* *} \mathrm{P}<0.001$, interference vs. blank control and empty vector. Lanes 1,2 and 3 represent blank control, empty vector and interference groups, respectively, in the SW626 cell line.

factor receptor (EGFR) protein may promote cell proliferation via activating the ERK1/2 signal pathway. In the present study, the effects of CXCR4-shRNA on ERK1/2 signaling in ovarian cancer cells were additionally investigated, and it was demonstrated that cells transfected with CXCR4-shRNA exhibited significantly decreased expression levels of ERK $1 / 2$, whereas no significant difference was identified between the empty vector group and the blank control. These results indicate that CXCR4-shRNA may decrease ERK1/2 protein expression and that such inhibition may be achieved by regulating EGFR expression to promote cancer cell apoptosis. 
ASK1 is a member of the MAPKKK family that performs significant functions in regulating cell apoptosis and differentiation, and in the immune response $(19,20)$. In the MAPK signaling pathway, ASK1 is located upstream of JNK and p38; as such, once it is activated, it may activate MAPKK and additionally JNK and p38, thereby causing cell apoptosis via mitochondria-associated caspase-3 (21). In normal cells, ASK1 activation is strictly controlled by the phosphorylation/dephosphorylation of threonine/serine or other protein-protein interactions. In breast cancer, increased ASK1 expression induced cell apoptosis (22), and subsequent experiments in human osteosarcoma cells additionally confirmed that the apoptosis-inducing factors exert their antitumor function through the proto-oncogene tyrosine-protein kinase reactive oxygen species/ASK1 pathway (23). The present study revealed that CXCR4-shRNA-transfected ovarian cancer cells exhibited significantly increased ASK1 mRNA expression; therefore, it is hypothesized that the transfection process activated ASK1 and c-Jun, thereby inducing the apoptosis of cancer cells.

The oncogene c-Jun encodes the Jun C protein, which may form homodimers with Jun B or Jun D, or even heterodimers with proteins from the Fos family. The homodimer or heterodimer formed by c-Jun or c-Fos proteins is termed activator protein 1 . The expression of c-Jun is regulated by a complex process that primarily involves JNK as a regulator, and transforming growth factor- $\beta$, bone morphogenetic proteins, hypoxia factors and oxidative stress as stimulators; c-Jun activation serves significant roles in cell proliferation, apoptosis, the stress response and tumor development $(24,25)$. The present study demonstrated that CXCR4-shRNA transfection increased p-c-Jun expression in ovarian cancer cells, indicating that CXCR4-shRNA may induce cancer cell apoptosis by regulating EGFR-mediated p-c-Jun expression.

The results of the present study suggest that silencing the $C X C R 4$ gene with shRNA inhibited proliferation and promoted apoptosis of epithelial ovarian cancer cells, and it was hypothesized that this was achieved via MAPK signaling, as decreased MAPK pathway activity was observed. These data provide a novel theoretical basis for investigating the mechanism by which CXCR4-shRNA promotes cell apoptosis, and lay a concrete foundation for use of the CXCR4 gene as a therapy target in the treatment of ovarian cancer.

\section{References}

1. Weiderpass E and Labreche F: Malignant tumors of the female reproductive system. Saf Health Work 3: 166-180, 2012.

2. Huang CY, Lee CY, Chen MY, Yang WH, Chen YH, Chang $\mathrm{CH}$, Hsu HC, Fong $\mathrm{YC}$ and Tang $\mathrm{CH}$ : Stromal cell-derived factor-1/CXCR4 enhanced motility of human osteosarcoma cells involves MEK1/2, ERK and NF-kappaB-dependent pathways. J Cell Physiol 221: 204-212, 2009.

3. Scotton CJ, Wilson JL, Milliken D, Stamp G and Balkwill FR: Epithelial cancer cell migration: A role for chemokine receptors? Cancer Res 61: 4961-4965, 2001.

4. Chen Y, Stamatoyannopoulos G and Song CZ: Down-regulation of CXCR4 by inducible small interfering RNA inhibits breast cancer cell invasion in vitro. Cancer Res 63: 4801-4804, 2003.

5. Chen HY, Wang JM, Wang HY, Zhang YX, Liu W, Pan L, Wang WH, Chen SF, Jin WG and Wang L: Effect of short hairpin RNA-induced CXCR4 silence on ovarian cancer cell. Biomed Pharmacother 66: 549-553, 2012.
6. Choi KC, Auersperg N and Leung PC: Mitogen-activated protein kinases in normal and (pre)neoplastic ovarian surface epithelium. Reprod Biol Endocrinol 1: 71, 2003.

7. Livak KJ and Schmittgen TD: Analysis of relative gene expression data using real-time quantitative PCR and the 2(-Delta Delta C(T)) method. Methods 25: 402-408, 2001.

8. Park J, Song KH and Ha H: Fractalkine increases mesangial cell proliferation through reactive oxygen species and mitogen-activated protein kinases. Transplant Proc 44: 1026-1028, 2012.

9. Yang SH, Sharrocks AD and Whitmarsh AJ: MAP kinase signalling cascades and transcriptional regulation. Gene 513: 1-13, 2013.

10. Janku F, Wheler JJ, Westin SN, Moulder SL, Naing A, Tsimberidou AM, Fu S, Falchook GS, Hong DS, Garrido-Laguna I, et al: PI3K/AKT/mTOR inhibitors in patients with breast and gynecologic malignancies harboring PIK3CA mutations. J Clin Oncol 30: 777-782, 2012.

11. Hayakawa J, Ohmichi M, Kurachi H, Ikegami H, Kimura A, Matsuoka T, Jikihara H, Mercola D and Murata Y: Inhibition of extracellular signal-regulated protein kinase or c-Jun N-terminal protein kinase cascade, differentially activated by cisplatin, sensitizes human ovarian cancer cell line. J Biol Chem 274: 31648-31654, 1999.

12. Yoshioka K: Scaffold proteins in mammalian MAP kinase cascades. J Biochem 135: 657-661, 2004.

13. Kim EK and Choi EJ: Pathological roles of MAPK signaling pathways in human diseases. Biochim Biophys Acta 1802: 396-405, 2010.

14. Zhao HB, Tang CL, Hou YL, Xue LR, Li MQ, Du MR and Li DJ: CXCL12/CXCR4 axis triggers the activation of EGF receptor and ERK signaling pathway in CsA-induced proliferation of human trophoblast cells. PLoS One 7: e38375, 2012.

15. Vergara D, Simeone P, Toraldo D, Del Boccio P, Vergaro V, Leporatti S, Pieragostino D, Tinelli A, De Domenico S, Alberti S, et al: Resveratrol downregulates Akt/GSK and ERK signalling pathways in OVCAR-3 ovarian cancer cells. Mol Biosyst 8: 1078-1087, 2012.

16. Porcile C, Bajetto A, Barbero S, Pirani P and Schettini G: CXCR4 activation induces epidermal growth factor receptor transactivation in an ovarian cancer cell line. Ann N Y Acad Sci 1030: 162-169, 2004.

17. Shen X, Wang S, Wang H, Liang M, Xiao L and Wang Z: The role of SDF-1/CXCR4 axis in ovarian cancer metastasis. J Huazhong Univ Sci Technolog Med Sci 29: 363-367, 2009.

18. Tarcic G and Yarden Y: MAP Kinase activation by receptor tyrosine kinases: In control of cell migration. Methods Mol Biol 661: 125-135, 2010.

19. Komuro Y, Takeda $\mathrm{K}$ and Ichijo H: Regulatiory mechanism of cell dealth through ASK1. Seikagaku 76: 1458-1462, 2004 (In Japanese).

20. Matsuzawa A and Ichijo H: Redox control of cell fate by MAP kinase: Physiological roles of ASK1-MAP kinase pathway in stress signaling. Biochim Biophys Acta 1780: $1325-1336,2008$

21. Hatai T, Matsuzawa A, Inoshita S, Mochida Y, Kuroda T, Sakamaki K, Kuida K, Yonehara S, Ichijo H and Takeda K: Execution of apoptosis signal-regulating kinase 1 (ASK1)-induced apoptosis by the mitochondria-dependent caspase activation. J Biol Chem 275: 26576-26581, 2000.

22. Guo Y, Xu X, Liu Z, Zhang T, Zhang X, Wang L, Wang M, Liu Y, Lu Y, Liu Y, et al: Apoptosis signal-regulating kinase 1 is associated with the effect of claudin-6 in breast cancer. Diagn Pathol 7: $111,2012$.

23. Chen JT, Fong YC, Li TM, Liu JF, Hsu CW, Chang CS and Tang CH: DDTD, an isoflavone derivative, induces cell apoptosis through the reactive oxygen species/apoptosis signal-regulating kinase 1 pathway in human osteosarcoma cells. Eur J Pharmacol 597: 19-26, 2008.

24. Qu WS, Tian DS, Guo ZB, Fang J, Zhang Q, Yu ZY, Xie MJ, Zhang HQ, Lu JG and Wang W: Inhibition of EGFR/MAPK signaling reduces microglial inflammatory response and the associated secondary damage in rats after spinal cord injury. J Neuroinflammation 9: 178, 2012.

25. Zhen YZ, Lin YJ, Gao JL, Zhao YF and Xu AJ: Rhein lysinate inhibits cell growth by modulating various mitogen-activated protein kinases in cervical cancer cells. Oncol Lett 2: 129-133, 2011. 